
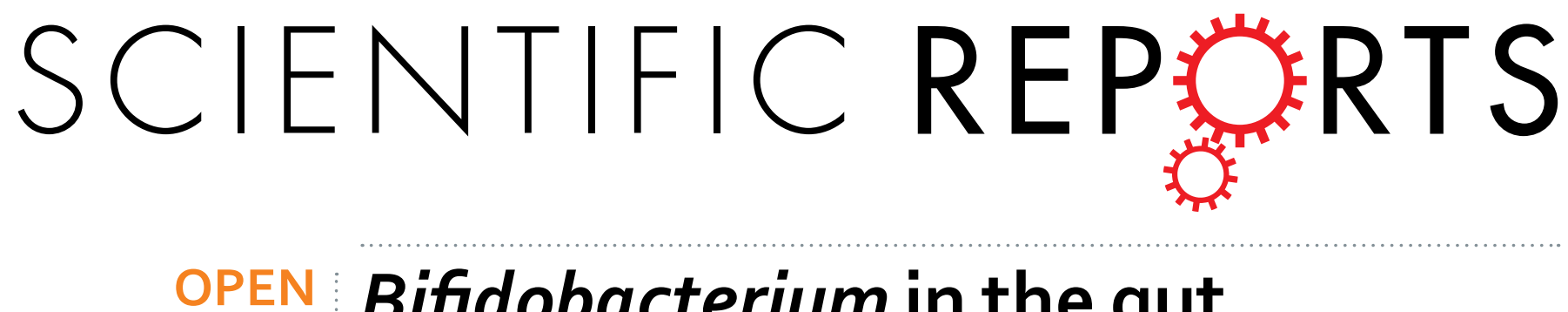

\title{
Bifidobacterium in the gut microbiota confer resilience to chronic social defeat stress in mice
}

Received: 23 January 2017

Accepted: 06 March 2017

Published: 03 April 2017

\author{
Chun Yang, Yuko Fujita, Qian Ren, Min Ma, Chao Dong \& Kenji Hashimoto
}

Accumulating evidence suggests that abnormalities in the composition of the gut microbiota may play a role in the pathogenesis of depression. Although approximately $30 \%$ mice are resilient to chronic social defeat stress (CSDS), the role of gut microbiota in this stress resilience is unknown. In this study, male C57BL/6 mice were exposed to a different CD1 aggressor mouse for 10 min on 10 consecutive days. A social interaction test was applied to distinguish between resilient and susceptible mice. Using $16 \mathrm{~S}$ rRNA analysis, we examined the composition of gut microbiota in feces from control, resilient, and susceptible mice. The marked appearance of Bifidobacterium was detected in the resilient mice, whereas in the control and susceptible mice, Bifidobacterium were below the detection limit. Oral intake of Bifidobacterium significantly increased the number of resilient mice after CSDS compared with vehicletreated mice. These findings suggest that Bifidobacterium may confer resilience to CSDS. Therefore, supplementation of Bifidobacterium may prevent the onset of depression from stress in humans. In addition, supplementation of Bifidobacterium may prevent or minimize relapse from remission induced by inflammation and/or stress in depressed patients.

Depression is a severe and chronic psychiatric disease affecting 350 million individuals worldwide. Indeed, approximately 1 million individuals commit suicide every year ${ }^{1}$. Humans display wide physiological variabilities in their response to stressors after exposure to psychological stress ${ }^{2}$. Several lines of evidence show that resilience is mediated by adaptive changes in several neural circuits, including numerous neurotransmitters and molecular pathways $^{3-5}$. However, the precise underlying mechanisms of stress resilience in psychiatric disorders, such as depression, remain obscure.

The gut-microbiota-brain axis consists of bidirectional communication between the gut and brain ${ }^{6,7}$. Accumulating evidence suggests that the brain acts on gastrointestinal and immune functions that have inherent assistance in shaping the gut's microbial composition ${ }^{8,9}$ and that gut microbes could affect host brain functions by producing and secreting substances consisting of neurotransmitters and metabolites ${ }^{10-12}$. Recent advancements in the alleviation of psychiatric diseases by optimizing the composition of the gut microbiota have attracted attention ${ }^{13,14}$. Dysbiosis in the gut may be implicated in the development or exacerbation of depression ${ }^{15}$. Antidepressants have pharmacological properties that exert beneficial effects by improving gut microbiota ${ }^{16-18}$.

We previously reported that neurological processes, including, glutamatergic and $\gamma$-aminobutyric acid (GABA) ergic neurotransmissions, brain-derived neurotrophic factor, dendritic spine density, peripheral interleukin-6 (IL-6), Keap1-Nrf2 system, and soluble epoxide hydrolase, may confer stress resilience in learned helplessness and chronic social defeat stress (CSDS) models ${ }^{19-24}$. However, there are no reports showing the role of gut microbiota in stress resilience.

Therefore, the present study was undertaken to examine whether the composition of gut microbiota in the feces of resilient and susceptible mice after CSDS is altered. The gram-positive bacteria Bifidobacterium were detected in resilient mice, but not in control or susceptible mice. Bifidobacterium are non-motile bacteria ${ }^{25}$ that have been well established as probiotic and ubiquitous inhabitants of the gastrointestinal tract, vagina, and mouth of humans ${ }^{26}$. Preclinical and clinical studies show that Bifidobacterium may have therapeutic benefits for mood disorders ${ }^{27-29}$. Therefore, we examined whether oral intake of Bifidobacterium could elicit a beneficial effect in the facilitation of stress resilience. 


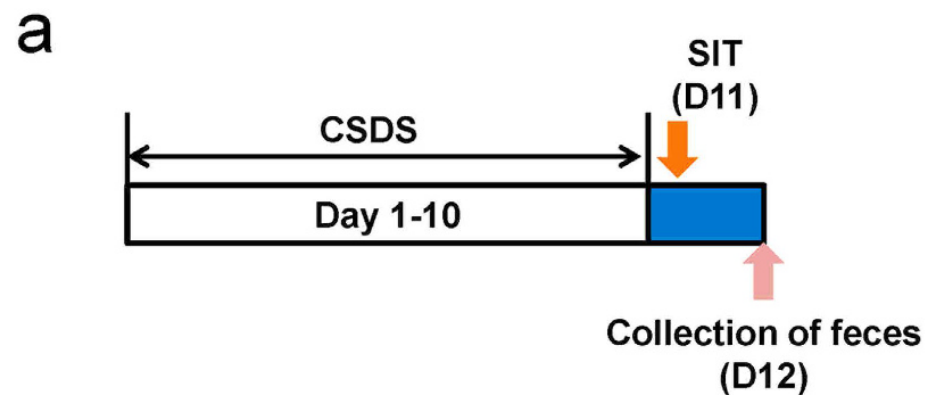

b
No target

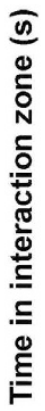

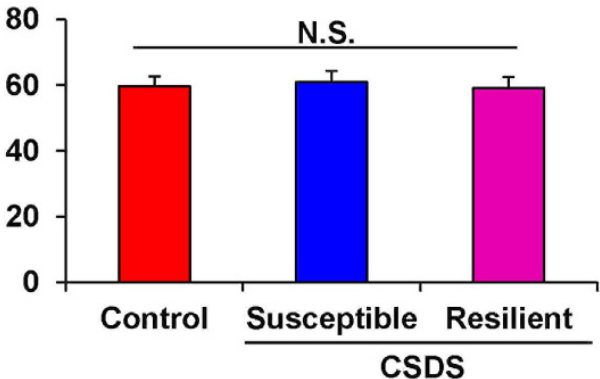

C

Target

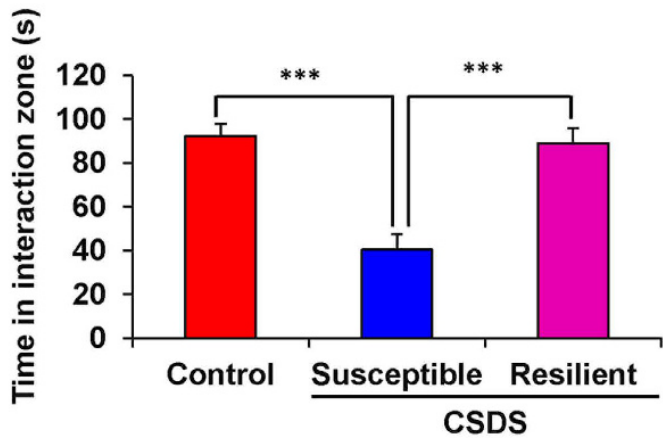

Figure 1. The duration the mice spent in the interaction zone of social interaction test. (a) The schedule of social defeat stress model (CSDS), social interaction test (SIT) and collection of feces. SIT was performed on day 11. Feces from all mice were collected on day 12. Control $(n=8)$, susceptible $(n=8)$, and resilient $(n=6)$ mice were used for subsequent analysis of composition of gut microbiota. (b) Duration of mice in the interaction zone without presence of aggressive CD1 mice. (c) Duration of mice in the interaction zone in the presence of aggressive CD1 mice. Data are shown as mean \pm S.E.M. ${ }^{* *} P<0.001$ compared to susceptible group of CSDS group. CSDS: chronic social defeat stress.

\section{Results}

Effects of CSDS on the duration time spent in the interaction area. CSDS is an animal model of depression using social conflicts between members of the same species to generate emotional and psychological stress $^{19,21}$. Here we successfully constructed the CSDS model according to our previous studies ${ }^{19,21}$. One day after CSDS, we divided susceptible and resilient mice by evaluating the time each mouse spent in the interaction area (Fig. 1a). The duration the mice spent in the interaction area in the absence of CD1 mice showed no statistical change $\left[F_{(2,19)}=0.079, P<0.925\right]$ (Fig. $1 \mathrm{~b}$ and Table $\mathrm{S} 1$ ). However, in the $2.5 \mathrm{~min}$ of the latter period of the social interaction test, susceptible mice significantly decreased their interaction time compared with control or resilient mice $\left[F_{(2,19)}=21.063, P<0.001\right]$ (Fig. 1c and Table S1).

Analysis of fecal bacteria. As described in the methods, susceptible mice $(<1)$ and resilient mice $(>1)$ were screened based on the interaction ratio of the social interaction test. Susceptible mice showed quite a distinct profile of fecal bacteria compared with control and resilient mice (Fig. 2). The emergence of fecal Bifidobacterium was observed in the resilient mice, but not in control or susceptible mice. The Fisher's exact test showed that the resilient mice had a significant increase in Bifidobacterium compared with control or susceptible mice $(P<0.01)$ (Fig. 3).

Effect of oral intake of Bifidobacterium on social interaction and sucrose preference tests. To examine the prophylactic effects of Bifidobacterium on susceptible mice after CSDS, Bifidobacterium or vehicle were injected orally into mice for 20 consecutive days (Fig. 4a). There were no changes in body weight among the four groups [time: $F_{(2,11)}=8.137, P=0.001$; treatment: $F_{(3,11)}=2.393, P=0.073$; and interaction (time $\times$ treatment): $F_{(6,11)}=1.027, P=0.413$ ] (Fig. $\left.4 \mathrm{~b}\right)$. In the absence of CD1 mice, the intake of Bifidobacterium failed to show a statistical change in the duration of time the mice spent in the interaction area among the four groups [group: $F_{(1,33)}=2.58, P=0.118$; treatment: $F_{(1,33)}=0.359, P=0.553$; interaction (group $\times$ treatment): $F_{(1,33)}=0.064$, $P=0.802$ ] (Fig. 4c and Table S2). However, in the presence of a CD1 mouse, two-way ANOVA showed that CSDS significantly decreased the duration of time the mice spent in the interaction area [group: $F_{(1,33)}=20.096$, $P<0.118$; treatment: $F_{(1,33)}=4.339, P=0.045$; interaction (group $\times$ treatment): $F_{(1,33)}=7.82, P=0.009$ ] (Fig. $4 \mathrm{~d}$ and Table S2).

The sucrose preference test has widely been used for the evaluation of anhedonia, which is a core symptom of depression ${ }^{30}$. Intake of Bifidobacterium for 20 consecutive days significantly attenuated the decreased sucrose 


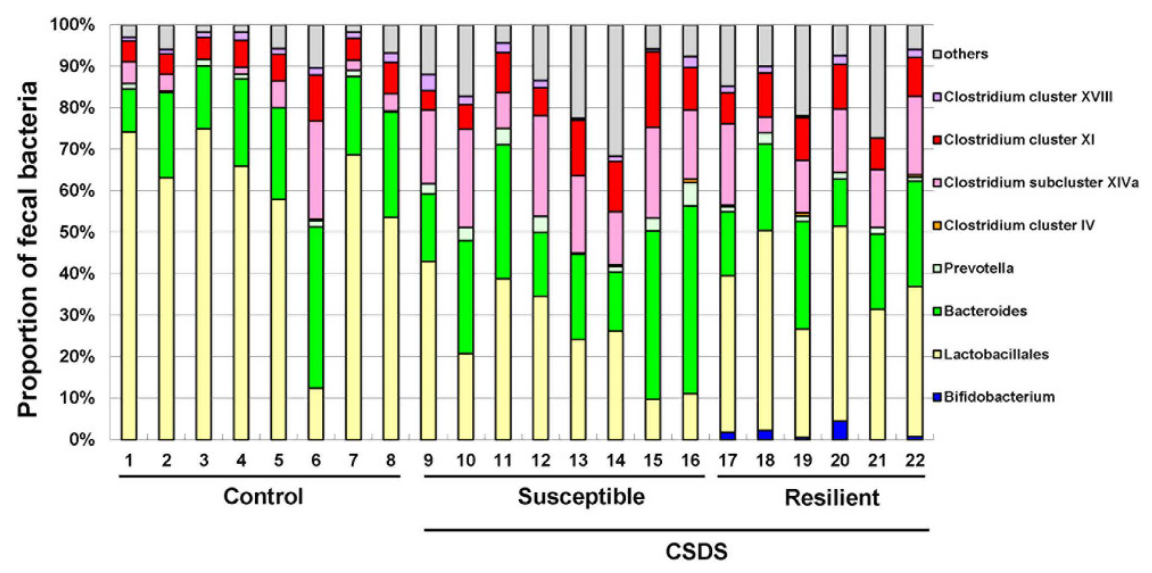

Figure 2. Analysis of fecal bacterium composition. Terminal Restriction Fragment Length Polymorphism (T-RFLP) was applied to analyze the fecal bacterium composition in control $(n=8)$, susceptible $(n=8)$ and resilient $(n=6)$ groups. CSDS: chronic social defeat stress.

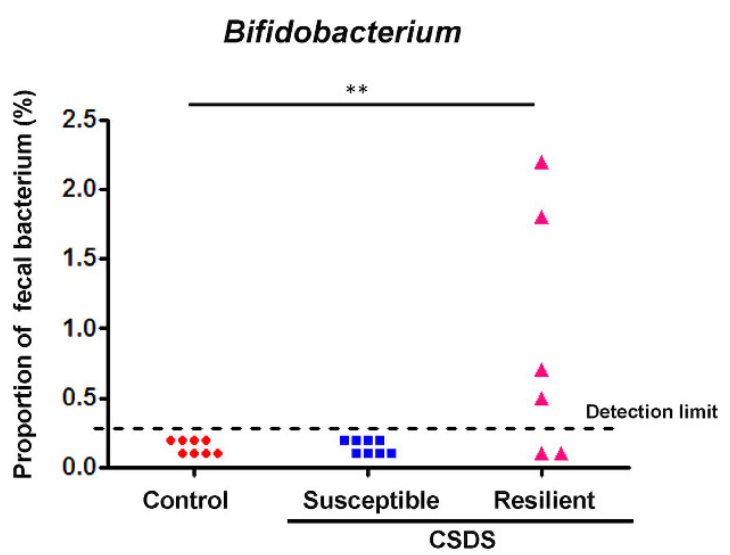

Figure 3. Fecal levels of Bifidobacterium in control, susceptible and resilient mice. Data are shown as the dot plot of each mouse (control group: $n=8$, susceptible group: $n=8$, resilient group: $n=6$ ). ${ }^{\star \star} P<0.01$ compared to control group (Fisher's exact test). CSDS: chronic social defeat stress.

preference in mice compared with that of mice with vehicle intake [group: $F_{(1,31)}=15.09, P<0.001$; treatment: $F_{(1,31)}=8.667, P=0.006$; interaction (group $\times$ treatment): $\left.F_{(1,31)}=16.491, P<0.001\right]$ (Fig. $\left.4 \mathrm{e}\right)$. These results suggest that the intake of Bifidobacterium may prevent the onset of depression-like phenotypes (susceptibility) in mice after CSDS.

\section{Discussion}

The major findings of this study are as follows: First, stress resilience after CSDS may be associated with the emergence of Bifidobacterium in the host gut. Second, oral intake of Bifidobacterium significantly increased the number of resilient mice after CSDS. These findings suggest that marked increases in Bifidobacterium may contribute to stress resilience after CSDS and that supplementation of Bifidobacterium may prevent the onset of depression-like phenotypes after CSDS.

Bifidobacterium, a genus of gram-positive anaerobic bacteria, are ubiquitous inhabitants of the gastrointestinal tract, vagina, and mouth ${ }^{25}$. Furthermore, Bifidobacterium are one of the major genera of Actinobacteria that make up the colon microbiota in mammals. During early infancy, Bifidobacterium may make up to $95 \%$ of the fecal flora of breast-fed babies. After weaning and subsequent exposure to food-derived and environmental microorganisms, the relative abundance of Bifidobacterium decreases. After establishment of the adult microbiota, the numbers of Bifidobacterium remain relatively stable at 3-6\% of all bacteria. Moreover, Bifidobacterium may produce short-chain fatty acids to decrease the gut $\mathrm{pH}$, form biological barriers, and secrete anti-microbial compounds to attenuate harmful bacteria ${ }^{31,32}$. Postnatal stress, such as mother-child separation, caused an altered composition of microbiota and decreased Bifidobacterium levels in the gut of rhesus monkeys ${ }^{33}$. A recent study showed that depressed patients have significantly lower fecal counts of Bifidobacterium than healthy controls, suggesting the role of Bifidobacterium in the pathogenesis of depression ${ }^{34}$. Collectively, these findings suggest that reduced composition of Bifidobacterium in the gut may accelerate the onset of depression.

In this study, we did not detect Bifidobacterium in control or susceptible mice because of the detection limits of the assay. In contrast, Friswell et al. ${ }^{35}$ reported the detection of Bifidobacterium in control C57BL/6 mice. 
a

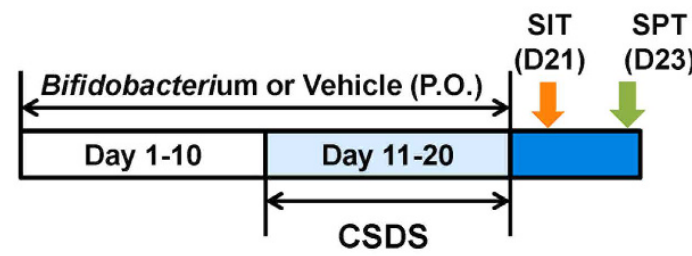

C

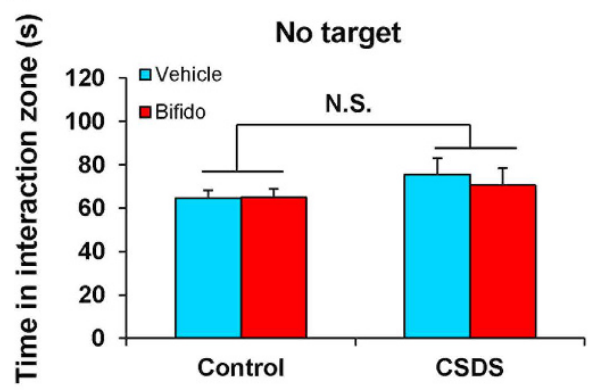

e

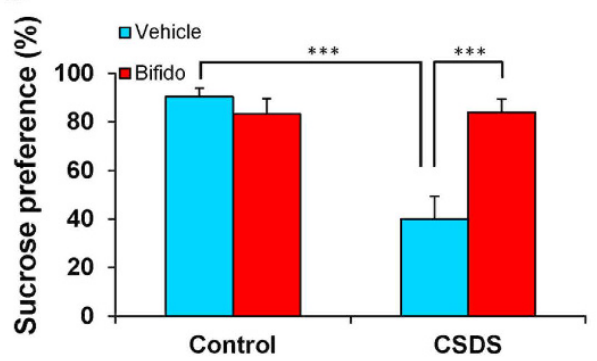

b
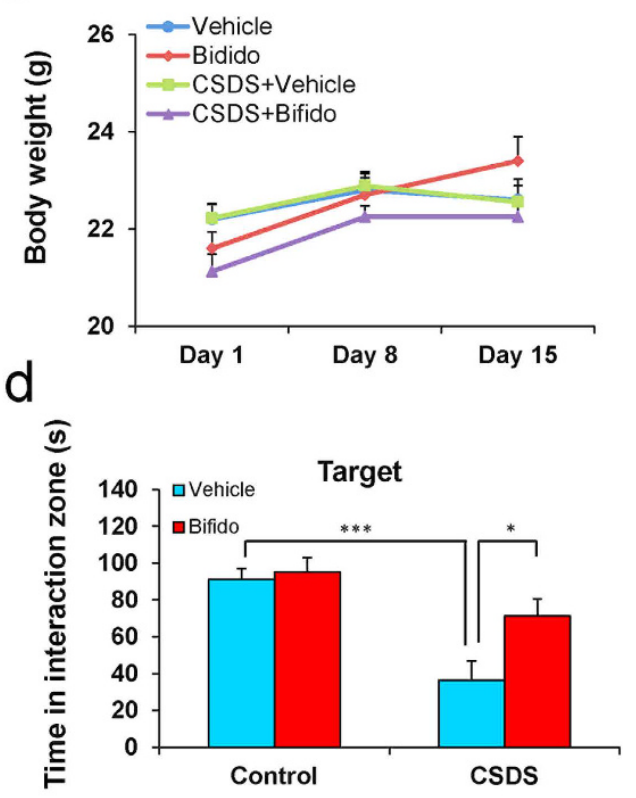

Figure 4. Effects of oral intake of Bifidobacterium on depression-like phenotype after CSDS. (a) The schedule of social defeat stress model (CSDS), social interaction test (SIT), sucrose preference test (SPT) and collection of feces. Bifidobacterium ( $10 \mathrm{mg} / \mathrm{kg} /$ day for 20 days) or vehicle ( $10 \mathrm{ml} / \mathrm{kg} /$ day for 20 days) were given orally from day 1 to day 20. CSDS was performed from day 11 to day 20. SIT and SPT were performed on day 21 and day 23, respectively. (b) Time course of body weight of mice. (c) Duration of mice in the interaction zone without presence of aggressive CD1 mice. (d) Duration of mice in the interaction zone in the presence of aggressive CD1 mice. (e) Sucrose preference test. Data are shown as mean \pm S.E.M. $(\mathrm{n}=8-10)$. ${ }^{\star} P<0.05$, ${ }_{* * *} P<0.001$ compared to vehicle-treated CSDS group.

Although the reason underlying this discrepancy is unclear, the method (terminal restriction fragment length polymorphism analysis) used in this study may contribute to the difference. We demonstrated marked increases in Bifidobacterium levels in resilient mice after CSDS, suggesting that marked increases in Bifidobacterium in the gut may play a role in stress resilience. Bifidobacterium are known to reduce intestinal endotoxin levels and improve mucosal barrier function. However, precise mechanisms underlying an increase in gut Bifidobacterium in resilient mice are currently unknown and further studies are needed.

Microbiota has a wide variety of physiological actions on the host ${ }^{36}$. Indeed, the crosstalk between the gut and brain is predominately influenced by the gut bacteria ${ }^{6-11}$. It is well established that an imbalance in gut microbiota can cause an abnormal gut-microbiota-brain axis resulting in several neurological and psychiatric diseases $^{6,7,37}$. Accumulating evidence suggests that dysfunction of the gut-microbiota-brain axis is of substantial relevance to mood disorders ${ }^{6,7,38}$. An unhealthy diet has emerged as one of the significant risk factor for dysbiosis that can induce the onset of depression ${ }^{38}$. Recent studies showed that gut microbiota may enhance the activity of CNS by producing and secreting neuroactive substances, such as serotonin and GABA ${ }^{39,40}$. Probiotics have antidepressant-like and anxiolytic activities in rodents ${ }^{41}$. The effects of probiotics are strongly related to the modulation of immune and neuroendocrine systems ${ }^{41}$. It is also recognized that Bifidobacterium are the main gut bacteria involved in the positive effects observed after prebiotic supplementation. Indeed, supplementation of Bifidobacterium has been associated with lower bacterial translocation and endotoxaemia, leading to a decrease in inflammatory cascade activation in several models of gut bacteria translocation ${ }^{42}$. Given the beneficial effects of Bifidobacterium to human health, it is likely that supplementation of Bifidobacterium may improve depressive symptoms or enhance stress resilience in humans. 
It has been well recognized that imbalanced inflammatory and anti-inflammatory responses are also involved in the pathogenesis of depression and targeted by the therapeutic effects of antidepressants ${ }^{43,44}$. Lipopolysaccharides (LPS), also known as lipoglycans and endotoxins, are the major component of the outer membrane of gram-negative bacteria, which has been verified to elicit several symptoms resembling depression ${ }^{45}$. Antidepressants not only have pharmacological benefits on depression but also have anti-inflammatory effects ${ }^{46}$. Elevated hypothalamic-pituitary-adrenal axis and depression-like behaviors in germ-free rats were successfully treated with Bifidobacterium ${ }^{47}$. Thus, treatment strategies targeting an increase in probiotics have led to beneficial actions on depressive symptoms and immune responses. Taken together, it is likely that supplementation of Bifidobacterium would have beneficial effects on the brain function by modifying the microbiota composition of the gut.

In conclusion, the present study suggests that increases in gut Bifidobacterium may contribute to stress resilience after CSDS. Given the role of gut Bifidobacterium in stress resilience, supplementation with Bifidobacterium may prevent the onset of depression from stress in humans. In addition, supplementation of Bifidobacterium may prevent or minimize relapse from remission induced by inflammation and/or stress in depressed patients.

\section{Materials and Methods}

Animals. Male adult C57BL/6 mice, aged 8 weeks (body weight 20-25g, Japan SLC, Inc., Hamamatsu, Japan) and male adult CD1 (ICR) mice, aged 13-15 weeks (body weight $>40$ g, Japan SLC, Inc., Hamamatsu, Japan) were used. Animals were housed under controlled temperatures and 12 hour light/dark cycles (lights on between 07:0019:00 h), with ad libitum food (CE-2; CLEA Japan, Inc., Tokyo, Japan) and water. This study was carried out in strict accordance with the recommendations in the Guide for the Care and Use of Laboratory Animals of the National Institutes of Health. The study was approved by the Chiba University Institutional Animal Care and Use Committee.

Materials. Bifidobacterium (LAC-B Granular Powder, Kowa Pharmaceutical, Ltd, Tokyo, Japan) was used in this study. The dose (10 mg/kg/day for 20 days) of Bifidobacterium or vehicle (water, $10 \mathrm{ml} / \mathrm{kg} /$ day for 20 days) was given into C57BL/6 mice (Fig. 4a).

Social defeat stress model. The procedure of social defeat stress was performed as previously reported ${ }^{19-21}$. Every day the C57BL/6 mice were exposed to a different CD1 aggressor mouse for $10 \mathrm{~min}$, total for 10 days. When the social defeat session ended, the resident CD1 mouse and the intruder mouse were housed in one half of the cage separated by a perforated Plexiglas divider to allow visual, olfactory, and auditory contact for the remainder of the 24 -h period. At $24 \mathrm{~h}$ after the last session, all mice were housed individually. On day 11, a social avoidance test was performed to identify subgroups of mice that were susceptible and unsusceptible to social defeat stress. This was accomplished by placing mice in an interaction test box $(42 \times 42 \mathrm{~cm})$ with an empty wire-mesh cage $(10 \times 4.5 \mathrm{~cm})$ located at one end. The movement of the mice was tracked for $2.5 \mathrm{~min}$, followed by $2.5 \mathrm{~min}$ in the presence of an unfamiliar aggressor confined in the wire-mesh cage. The duration of the subject's presence in the "interaction zone" (defined as the 8-cm-wide area surrounding the wiremesh cage) was recorded by a stopwatch. The interaction ratio was calculated as time spent in an interaction zone with an aggressor/time spent in an interaction zone without an aggressor. An interaction ratio of 1 was set as the cutoff: mice with scores $<1$ were defined as "susceptible" to social defeat stress and those with scores $\geq 1$ were defined as "unsusceptible".

Sucrose preference test (SPT). Mice were exposed to water and $1 \%$ sucrose solution for $48 \mathrm{~h}$, followed by 4 hours of water and food deprivation and a 1 hour exposure to two identical bottles, one is water, and another is $1 \%$ sucrose solution. The bottles containing water and sucrose were weighed before and at the end of this period and the sucrose preference was determined.

Fecal bacteria analysis. Fecal samples were collected after behavioral tests. The samples were stored in a refrigerator with a temperature of $-80^{\circ} \mathrm{C}$. The analysis of fecal bacteria was performed at TechnoSuruga Laboratory Co, Ltd. (Shizuoka, Japan). DNA was automatically extracted from the processed supernatant with the use of a 12GC and GC series Magtration-MagaZorb DNA Common Kit 200N (Precision System Science, Chiba, Japan). The final concentration of the extracted DNA was adjusted to $10 \mathrm{ng} / \mu \mathrm{L}$. Terminal restriction fragment length polymorphism (T-RFLP) analysis was performed according to the previous study ${ }^{48}$. The 16S rRNA gene was amplified with the use of primer sets 516F ( $5^{\prime}$-TGCCAGCAGCCGCGGTA- $3^{\prime}$ ) and 1492R (5'-GGTTACCTTGTTACGACTT-3'). The $5^{\prime}$-end of the forward primer 516F was labeled with $6^{\prime}$-carboxyfluorescein. Amplified polymerase chain reaction (PCR) products were refined with the usage of MultiScreen ${ }^{\circledR}$ PCR 96 filter plates (Millipore, Tokyo, Japan). The refined products (about $3 \mu \mathrm{L}$ ) were digested for $3 \mathrm{~h}$ at $55^{\circ} \mathrm{C}$ with $10 \mathrm{U}$ of $B s l$ I restriction enzyme (New England Biolabs, Inc., Ipswich, MA, USA). The length of the separated fluorescent PCR fragment was determined with an ABI PRISM 3130xl genetic analyzer (Applied Biosystems, Tokyo, Japan), and the data were analyzed with GeneMapper ${ }^{\circledR}$ software. MapMarker ${ }^{\circledR}$ X-Rhodamine Labeled 50-1000 bp (BioVentures, Inc., Murfreesboro, TN, USA) was used as a size standard marker. NTSYSpc software (Exeter Software, Setauket, NY, USA) was used to perform cluster analysis. Each terminal restriction fragment (T-RF) was expressed as a percentage of the peak area of all T-RFs. Disparity in similarity among fecal samples in individual mice was calculated using a correlation matrix and was presented graphically on tree diagrams with the use of a weighted pair-group method with arithmetic mean (WPGMA) clustering ${ }^{48}$.

Statistical Analysis. The data are shown as the mean \pm standard error of the mean (S.E.M.). Analysis was performed by using PASW Statistics 20 (formerly SPSS statistics; SPSS, Tokyo, Japan). Comparisons between groups were performed by one-way or two-way analysis of variance (ANOVA), followed by post-hoc Tukey test. Fisher's exact test was used for comparison of proportion of fecal bacteria among the groups. Data of body weight were analyzed by repeated two-way ANOVA, followed by post-hoc Tukey test. The $P<0.05$ was considered statistically significant. 


\section{References}

1. World Health Organization (WHO) Depression. Fact sheet No. 369/October 2012. [(accessed on 10 March 2015)]. Available online: http://www.who.int/mediacentre/factsheets/fs369/en/index.html.

2. Beery, A. K. \& Kaufer, D. Stress, social behavior, and resilience: insights from rodents. Neurobiol Stress 1, 116-127 (2015).

3. Russo, S. J., Murrough, J. W., Han, M. H., Charney, D. S. \& Nestler, E. J. Neurobiology of resilience. Nat Neurosci 15, 1475-1484 (2012).

4. Franklin, T. B., Saab, B. J. \& Mansuy, I. M. Neural Mechanisms of Stress Resilience and Vulnerability. Neuron 75, 747-761 (2012).

5. Russo, S. J. \& Nestler, E. J. The brain reward circuitry in mood disorders. Nat Rev Neurosci 14, 609-625 (2013).

6. Kelly, J. R., Clarke, G., Cryan, J. F. \& Dinan, T. G. Brain-gut-microbiota axis: challenges for translation in psychiatry. Ann Epidemiol 26, 366-372 (2016)

7. Fung, T. C., Olson, C. A. \& Hsiao, E. Y. Interactions between the microbiota, immune and nervous systems in health and disease. Nat Neurosci 20, 145-155 (2017).

8. Carabotti, M., Scirocco, A., Maselli, M. A. \& Severi, C. The gut-brain axis: interactions between enteric microbiota, central and enteric nervous systems. Ann Gastroenterol 28, 203-209 (2015).

9. Ha, C. W., Lam, Y. Y. \& Holmes, A. J. Mechanistic links between gut microbial community dynamics, microbial functions and metabolic health. World J Gastroenterol 20, 16498-16517 (2014).

10. Kiraly, D. D. et al. Alterations of the host microbiome affect behavioral responses to cocaine. Sci Rep 6, 35455 (2016).

11. Borrelli, L. et al. Probiotic modulation of the microbiota-gut-brain axis and behaviour in zebrafish. Sci Rep 6, 30046 (2016).

12. Smith, P. A. The tantalizing links between gut microbes and the brain. Nature 526, 312-314 (2015).

13. O'Mahony, S. M., Clarke, G., Borre, Y. E., Dinan, T. G. \& Cryan, J. F. Serotonin, tryptophan metabolism and the brain-gutmicrobiome axis. Behav Brain Res 277, 32-48 (2015).

14. Neufeld, K. A. \& Foster, J. A. Effects of gut microbiota on the brain: implications for psychiatry. J Psychiatry Neurosci 34, 230-231 (2009).

15. Desbonnet, L. et al. Gut microbiota depletion from early adolescence in mice: Implications for brain and behaviour. Brain Behav Immun 48, 165-173 (2015).

16. Rogers, G. B., Keating, D. J., Young, R. L., Wong, M. L., Licinio, J. \& Wesselingh, S. From gut dysbiosis to altered brain function and mental illness: mechanisms and pathways. Mol Psychiatry 21, 738-748 (2016).

17. Cryan, J. F. \& Dinan, T. G. Mind-altering microorganisms: the impact of the gut microbiota on brain and behaviour. Nat Rev Neurosci 13, 701-712 (2012).

18. Macedo, D. et al. Antidepressants, antimicrobials or both? Gut microbiota dysbiosis in depression and possible implications of the antimicrobial effects of antidepressant drugs for antidepressant effectiveness. J Affect Disord 208, 22-32 (2016).

19. Yao, W. et al. Role of Keap1-Nrf2 signaling in depression and dietary intake of glucoraphanin confers stress resilience in mice. Sci Rep 6, 30659 (2016)

20. Yang, B. et al. Regional differences in the expression of brain-derived neurotrophic factor (BDNF) pro-peptide, proBDNF and preproBDNF in the brain confer stress resilience. Eur Arch Psychiatry Clin Neurosci 266, 765-769 (2016).

21. Ren, Q. et al. Gene deficiency and pharmacological inhibition of soluble epoxide hydrolase confers resilience to repeated social defeat stress. Proc Natl Acad Sci USA 113, E1944-52 (2016).

22. Yang, C., Shirayama, Y., Zhang, J. C., Ren, Q. \& Hashimoto, K. Peripheral interleukin-6 promotes resilience versus susceptibility to inescapable electric stress. Acta Neuropsychiatr 27, 312-6 (2015).

23. Yang, C., Shirayama,Y., Zhang,J. C., Ren,Q. \& Hashimoto, K. Regional differences in brain-derived neurotrophic factor levels and dendritic spine density confer resilience to inescapable stress. Int J Neuropsychopharmacol 18, pyu121 (2015).

24. Muneoka, K., Shirayama, Y., Horio, M., Iyo, M. \& Hashimoto, K. Differential levels of brain amino acids in rat models presenting learned helplessness or non-learned helplessness. Psychopharmacology (Berl) 229, 63-71 (2013).

25. Duranti, S. et al. Evaluation of genetic diversity among strains of the human gut commensal Bifidobacterium adolescents. Sci Rep 6, 23971 (2016)

26. Kibe, R. Upregulation of colonic luminal polyamines produced by intestinal microbiota delays senescence in mice. Sci Rep 4 , 4548 (2014).

27. Messaoudi, M. et al. Assessment of psychotropic-like properties of a probiotic formulation (Lactobacillus helveticus R0052 and Bifidobacterium longum R0175) in rats and human subjects. Br J Nutr 105, 755-764 (2011).

28. Savignac, H. M., Kiely, B., Dinan, T. G. \& Cryan, J. F. Bifidobacteria exert strain-specific effects on stress-related behavior and physiology in BALB/c mice. Neurogastroenterol Motil 26, 1615-1527 (2014).

29. Desbonnet, L. et al. Effects of the probiotic Bifidobacterium infantis in the maternal separation model of depression. Neuroscience 170, 1179-1188 (2010).

30. Krishnan, V. \& Nestler, E. J. Animal models of depression: molecular perspectives. Curr Top Behav Neurosci 7, 121-147 (2011).

31. Bottacini, F., Ventura, M., van Sinderen, D. \& O'Connell Motherway, M. Diversity, ecology and intestinal function of bifidobacteria. Microb Cell Fact 13, S4 (2014).

32. Liao, Z. L. et al. Impact of the consumption of tea polyphenols on early atherosclerotic lesion formation and intestinal Bifidobacteria in high-fat-fed ApoE-/- mice. Front Nutr 3, 42 (2016).

33. Dinan, T. G. \& Cryan, J. F. Regulation of the stress response by the gut microbiota: implications for psychoneuroendocrinology. Psychoneuroendocrinology 37, 1369-1378 (2012).

34. Aizawa, E. et al. Possible association of Bifidobacterium and Lactobacillus in the gut microbiota of patients with major depressive disorder. J Affect Disord 202, 254-257 (2016).

35. Friswell, M. K. et al. Site and strain-specific variation in gut microbiota profiles and metabolism in experimental mice. PLoS One 5, e8584 (2010).

36. Ouwehand, A. C., Salminen, S. \& Isolauri, E. Probiotics: an overview of beneficial effects. Antonie Van Leeuwenhoek 82, 279-289 (2002).

37. Fond, G. et al. The "psychomicrobiotic": Targeting microbiota in major psychiatric disorders: A systematic review. Pathol Biol (Paris) 63, 35-42 (2015).

38. Foster, J. A. \& McVey Neufeld, K. A. Gut-brain axis: how the microbiome influences anxiety and depression. Trends Neurosci 36, 305-312 (2013).

39. Sekirov, I., Russell, S. L., Antunes, L. C. \& Finlay, B. B. Gut microbiota in health and disease. Physiol Rev 90, 859-904 (2010).

40. Yano J. M. et al. Indigenous bacteria from the gut microbiota regulate host serotonin biosynthesis. Cell 161, 264-276 (2015).

41. Dinan, T. G., Stanton, C. \& Cryan, J. F. Psychobiotics: a novel class of psychotropic. Biol Psychiatry 74, 720-726 (2013).

42. Caplan, M. S. et al. Bifidobacterial supplementation reduces the incidence of necrotizing enterocolitis in a neonatal rat model. Gastroenterology 117, 577-583 (1999).

43. Zhang, J. C., Yao, W. \& Hashimoto, K. Brain-derived neurotrophic factor (BDNF)-TrkB signaling in inflammation-related depression and potential therapeutic targets. Curr Neuropharmacol 14, 721-731 (2016).

44. Hashimoto, K. Inflammatory biomarkers as differential predictors of antidepressant response. Int J Mol Sci 16, 7796-7801 (2015).

45. Dantzer, R., O’Connor, J. C., Freund, G. G., Johnson, R. W. \& Kelley, K. W. From inflammation to sickness and depression: when the immune system subjugates the brain. Nat Rev Neurosci 9, 46-56 (2008). 
46. Konsman, J. P., Parnet, P. \& Dantzer, R. Cytokine-induced sickness behaviour: mechanisms and implications. Trends Neurosci 25, 154-159 (2002)

47. Messaoudi, M., Violle, N., Bisson, J. F., Desor, D., Javelot, H. \& Rougeot, C. Beneficial psychological effects of a probiotic formulation (Lactobacillus helveticus R0052 and Bifidobacterium longum R0175) in healthy human volunteers. Gut Microbes 2, 256-261 (2011).

48. Nagashima, K., Hisada, T., Sato, M. \& Mochizuki, J. Application of new primer-enzyme combinations to terminal restriction fragment length polymorphism profiling of bacterial populations in human feces. Appl Environ Microbiol 69, 1251-1262 (2003).

\section{Acknowledgements}

This study was partly supported by the Strategic Research Program for Brain Sciences from Japan Agency for Medical Research and Development, AMED (to K.H.). Dr. Chun Yang and Dr. Qian Ren were supported by Research Fellowship of the Japan Society for the Promotion of Science (Tokyo, Japan).

\section{Author Contributions}

K.H. designed the study and wrote the protocol. C.Y., Y.F., Q.R., M.M., and C.D. performed all the pharmacological experiments. C.Y. undertook the statistical analysis. C.Y. and K.H. wrote the first draft of the manuscript. All authors contributed to and have approved the final manuscript.

\section{Additional Information}

Supplementary information accompanies this paper at http://www.nature.com/srep

Competing Interests: Dr. Hashimoto has received research support from Dainippon Sumitomo, Mochida, Otsuka, and Taisho. Other authors state no potential conflicts of interest.

How to cite this article: Yang, C. et al. Bifidobacterium in the gut microbiota confer resilience to chronic social defeat stress in mice. Sci. Rep. 7, 45942; doi: 10.1038/srep45942 (2017).

Publisher's note: Springer Nature remains neutral with regard to jurisdictional claims in published maps and institutional affiliations.

(c) This work is licensed under a Creative Commons Attribution 4.0 International License. The images
or other third party material in this article are included in the article's Creative Commons license, unless indicated otherwise in the credit line; if the material is not included under the Creative Commons license, users will need to obtain permission from the license holder to reproduce the material. To view a copy of this license, visit http://creativecommons.org/licenses/by/4.0/

(C) The Author(s) 2017 